\title{
A fatal interstitial lung disease in an anti-melanoma differentiation-associated gene 5 (anti-MDA5) antibody negative patient with juvenile dermatomyositis
}

\author{
Osman Yeşilbaş ${ }^{1 \oplus}$, Mehmet Yıldız ${ }^{2 \oplus}$, Can Yılmaz Yozgat $^{3 \oplus}$, Irmak Tahaoğlu ${ }^{4 \oplus}$, \\ Hakan Yazan ${ }^{5 \oplus}$, Erkan Çakır ${ }^{5 \oplus}$, Amra Adrovic ${ }^{2 \oplus}$, Sezgin Şahin ${ }^{2 \oplus}$, Kenan Barut ${ }^{2 \oplus}$, \\ Özgür Kasapçopur ${ }^{20}$
}

Departments of ${ }^{1}$ Pediatric Critical Care Medicine, ${ }^{4}$ Pediatrics and ${ }^{5}$ Pediatric Pulmonology, ${ }^{3}$ Medical Student, Bezmialem Vakuf University Faculty of Medicine, İstanbul; ${ }^{2}$ Department of Pediatric Rheumatology, İstanbul University Cerrahpaşa Medical School, Istanbul, Turkey.

\begin{abstract}
Background. Juvenile dermatomyositis associated interstitial lung disease, rarely seen in pediatric age groups, has adverse effects on survival. Anti-melanoma differentiation associated gene 5, one of the identified autoantibodies in juvenile dermatomyositis, preferentially affects the lung tissue and may cause rapidly progressive interstitial lung disease. It is a major cause of mortality in juvenile dermatomyositis. In this case report, we present a pediatric patient diagnosed with juvenile dermatomyositis without anti-melanoma differentiation associated gene 5 antibody positivity.
\end{abstract}

Case. A six-year-old male patient admitted to the Pediatric Intensive Care Unit with symptoms of respiratory failure, 1.5 months after the diagnosis of juvenile dermatomyositis. Thorax computed tomography examination revealed pneumomediastinum, a trace of left-sided pneumothorax, atelectasis on the left posterior lung region, ground-glass opacity, minimal subpleural patchy consolidation, and subcutaneous emphysema especially on the sides of the chest wall. Broad-spectrum antibiotics were started. His nasal swab sample was positive in terms of influenza B; therefore, oseltamivir was added to the treatment. Autoimmune myositis antibodies panel was examined but all of them including anti-melanoma differentiation associated gene 5 antibody resulted as negative. There was no notable reduction in lung infiltrations with the patient's current treatment regimen. On the $12^{\text {th }}$ day of Pediatric Intensive Care Unit admission, thorax computed tomography scan revealed progressed radiological lung findings compatible with rapidly progressive interstitial lung disease secondary to juvenile dermatomyositis. Despite intensive medical and extracorporeal treatments such as pulse steroid, intravenous immunoglobulin, methotrexate, cyclophosphamide, rituximab, therapeutic plasma exchange and, extracorporeal membrane oxygenation, the patient died on the $35^{\text {th }}$ day.

Conclusions. Juvenile dermatomyositis patients should be carefully monitored for the development of interstitial lung disease. Rapidly progressive interstitial lung disease with a high mortality may develop shortly after diagnosis, even if the anti-melanoma differentiation associated gene 5 antibody is negative.

Key words: anti-melanoma differentiation associated gene 5, child, juvenile dermatomyositis, interstitial lung disease, rapidly progressive interstitial lung disease.

Juvenile dermatomyositis (JDM), one of the juvenile-onset myositis, is a very rare systemic autoimmune muscle disease and vasculopathies

Osman Yeşilbaş

drosmanyesilbas@gmail.com

Received 21st July 2020, revised 4th October 2020, accepted 23rd December 2020. of unknown etiology accompanied by characteristic skin manifestations such as Gottron's papules or periorbital heliotrope rash. It also has systemic manifestations, such as Raynaud's syndrome, arthritis, cardiac dysfunction, dysphagia, and various forms of pulmonary disease. ${ }^{1-3}$ Interstitial lung disease (ILD), the most common form of lung 
involvement, has adverse effect on survival. Anti-melanoma differentiation associated gene 5 (anti-MDA5), one of the identified autoantibodies in DM with an incidence of $7-38 \%$, preferentially affects the lung tissue and may cause rapidly progressive ILD (RP-ILD). It is a major cause of mortality in both adult and juvenile DM patients, particularly in East-Asian cohorts. ${ }^{2,3}$

In this case report, we present a very interesting pediatric patient diagnosed with juvenile DM (JDM) without anti-MDA5 antibody positivity. The disease has progressed to RPILD emerged with spontaneous pneumothorax and pneumomediastinum in a short time after diagnosis and our patient died despite intensive immunosuppressive and extracorporeal treatments such as therapeutic plasma exchange (TPE) and extracorporeal membrane oxygenation (ECMO).

\section{Case Report}

A six-year-old male patient was admitted to the Pediatric Intensive Care Unit (PICU) with symptoms of respiratory failure. The patient was conscious and had a Glasgow Coma Score of 15 . His vital signs were: blood pressure 106/65 $\mathrm{mmHg}$, pulse rate $140 / \mathrm{min}$., respiratory rate $36 /$ min., body temperature $37^{\circ} \mathrm{C}$. He had subcostal/ intercostal retractions and nasal flaring. Lung auscultation revealed bilateral crackles. Also, he had subcutaneous emphysema on his neck and chest, and heart auscultation revealed deep heart sounds. His oxygen saturation was between $80 \%$ and $85 \%$ in room air; thus, noninvasive ventilation (NIV) with high flow nasal cannula (HFNC) was started. It was learned that he had been diagnosed with JDM approximately one and half months ago with signs and symptoms of typical Gottron's papules (Fig. 1A) and periorbital heliotrope rash, fatigue, bilaterally distal and proximal interphalangeal arthritis (Fig. 1A), and oral aphthae (Fig. 1B). Hydroxychloroquine, prednisolone, and methotrexate had been started for JDM therapy.

On the PICU admission, blood gas, serum biochemistry, and electrolytes were within the normal range. C-reactive protein and procalcitonin levels were negative. On chest X-ray, there were bilateral infiltrates, subcutaneous emphysema on the sidewalls of the chest and neck without any signs of pneumothorax (Fig. 2). Thorax computed tomography (CT) examination revealed pneumomediastinum, a trace of left-sided pneumothorax, atelectasis on the left posterior lung region, ground-glass opacity, minimal subpleural patchy consolidation, and subcutaneous emphysema especially on the sides of the chest wall (Fig. 3).

Since the patient was still on immunosuppressive therapy, we decided to continue with

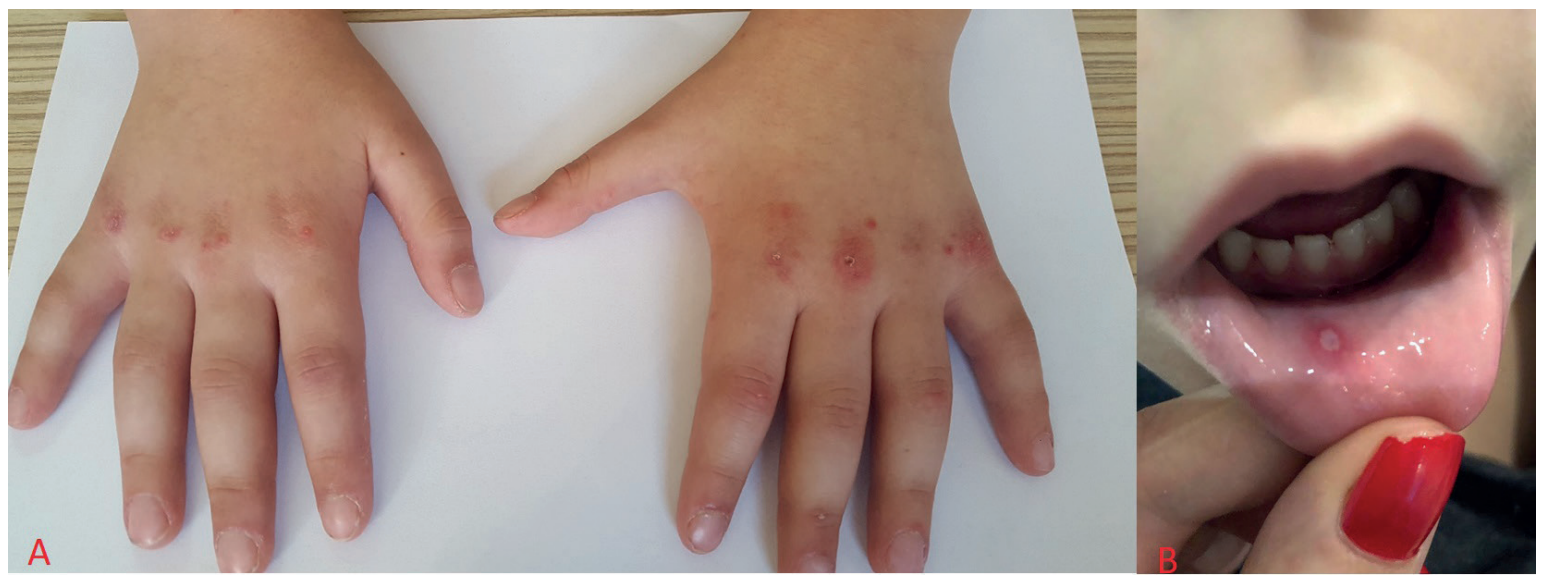

Fig. 1. A. Typical Gottron's papules and bilaterally distal and proximal interphalangeal arthritis. B. The patient's oral aphthae. 


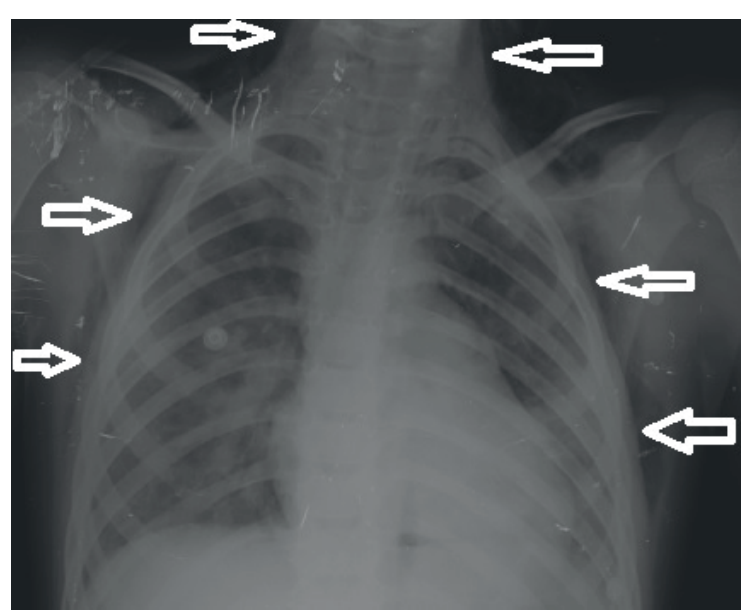

Fig. 2. Bilateral infiltrates, subcutaneous emphysema on the sidewalls of the chest and neck (white arrows).

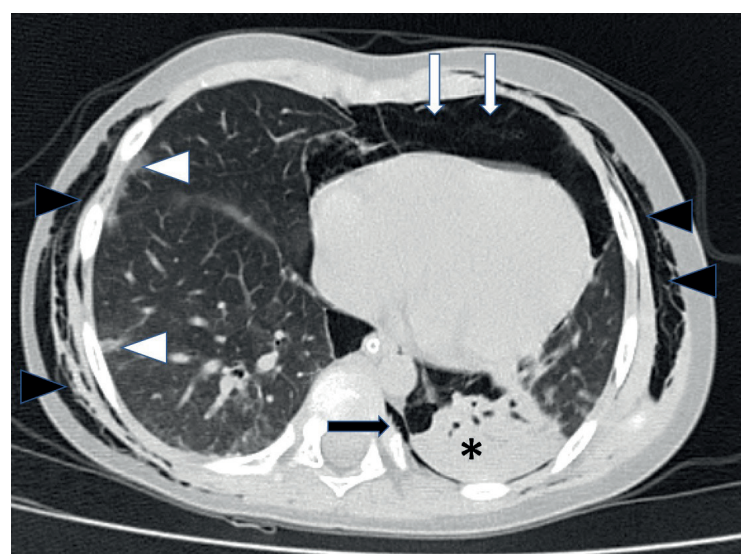

Fig. 3. First thorax computed tomography examination; pneumomediastinum (white arrows), a trace of left-sided pneumothorax (black arrow), atelectasis on the left posterior lung region (asterisk), ground-glass opacity, minimal subpleural patchy consolidation (white arrowheads), and subcutaneous emphysema especially on the lateral sides of the chest wall (black arrowheads).

intravenous (IV) piperacillin-tazobactam, teicoplanin, fluconazole, trimethoprimsulfamethoxazole and enterally azithromycin treatments which were started in the external center prior to the PICU admission. The virus panel examination, which was done by polymerase chain reaction (PCR) of the patient's nasal swab sample, was positive in terms of influenza B; therefore, oseltamivir (2x60 mg) was added to the treatment and teicoplanin was terminated. A tube was placed in the mediastinum and left chest under operating room conditions. Before the procedure, he was intubated and after the procedure he was started on invasive mechanical ventilator support in the PICU. A transthoracic echocardiographic examination revealed normal heart functions. Hydroxychloroquine and prednisolone were discontinued and methylprednisolone $(2 \mathrm{mg} /$ $\mathrm{kg} /$ day) was initiated. Autoimmune myositis antibodies panel (anti-MDA5, anti PM-Scl, anti Jo-1, anti $K u$, anti $P L-7$, anti $P L-12$, antiNXP2, anti-EJ, anti-SRP, anti MI2 alfa, anti MI2 beta, anti-SAE1, anti-TIF1g, anti-PM/ scl75, anti-OJ antibodies) was examined but all resulted as negative. Five days later, the patient was extubated and NIV was started with HFNC. Fluconazole and trimethoprimsulfamethoxazole treatments were terminated. Three days later, body temperature and acute phase reactants increased, new lung infiltrations occurred, and he was reintubated. Teicoplanin and trimethoprim-sulfamethoxazole treatments were restarted, and piperacillin-tazobactam treatment was replaced by meropenem. We decided to terminate methylprednisolone treatment by gradually decreasing it due to the patient's lung infection. The tests which were done especially for tuberculosis and immunodeficiencies were negative. There was no notable reduction in lung infiltrations with the patient's current treatment regimen. Galactomannan antigen, cytomegalovirus PCR, and all the cultures resulted as negative. On the 12th day of the PICU admission, thorax CT scan revealed progressed radiological lung findings compatible with RP-ILD secondary to JDM (Fig. 4); thus, five-day pulse steroid (30 $\mathrm{mg} / \mathrm{kg} /$ day), IV immunoglobulin (IVIG, $2 \mathrm{~g} / \mathrm{kg}$ ), and methotrexate (15 $\mathrm{mg} / \mathrm{m}^{2} /$ week) were started. Laboratory tests (anti-nuclear antibody, anti-dsDNA, ENA panel, $\mathrm{C}_{3^{\prime}} \mathrm{C}_{4^{\prime}}$ p-ANCA, c-ANCA, lupus anticoagulant, anticardiolipin, antiphospholipid, and antiglomerular basement membrane antibodies) for other concomitant vasculitis and rheumatic diseases were negative. Fluconazole was added to the patient's treatment for possible fungal pneumonia. On the $18^{\text {th }}$ day, the patient had no 


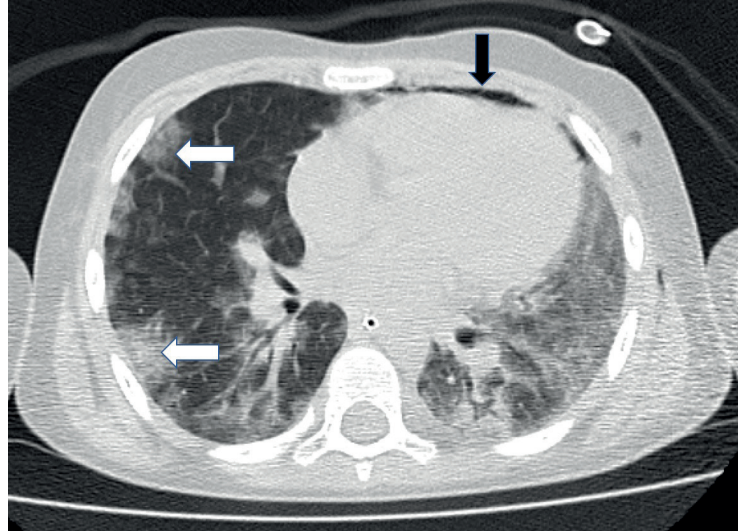

Fig. 4. Second thorax computed tomography examination; minimal pneumomediastinum (black arrow), ground-glass opacity, progressed subpleural patchy consolidation (white arrows), and widespread nonspecific interstitial pneumonia.

improvement in lung findings; therefore, high dose IV cyclophosphamide treatment (1000 $\mathrm{mg} / \mathrm{m} 2 / \mathrm{month}$ ) and daily TPE were started for RP-ILD secondary to JDM. Despite all these intensive treatments, hypoxia worsened, severe pediatric acute respiratory distress syndrome (PARDS) was diagnosed, and venoarterial ECMO was promptly initiated on the $20^{\text {th }}$ day of PICU admission. On the $23^{\text {rd }}$ day of PICU admission, rituximab $\left(375 \mathrm{mg} / \mathrm{m}^{2}\right)$ was started as a rescue therapy for RP-ILD and TPE terminated but the patient, who did not respond to any of these advanced medical and extracorporeal treatments, died on the $35^{\text {th }}$ day due to multiple organ failure.

Informed consent was received from the family.

\section{Discussion}

Lung involvement secondary to JDM is very rare in the pediatric age group. In our previous study, a total of 50 patients with JDM were reviewed retrospectively and none of our patients had lung involvement. ${ }^{4}$ In all age groups, the most common lung involvement in DM and polymyositis (PM) is ILD, and its prevalence rate is reported to be $23-65 \%$. Nonspecific interstitial pneumonia, organizing pneumonia/bronchiolitis, obliterans organizing pneumonia, usual interstitial pneumonia, diffuse alveolar damage, and pulmonary capillaritis are other forms of ILD. ${ }^{1}$ RP-ILD is uncommon, but it is still one of the significant causes of death in JDM. ${ }^{3,4}$ Kobayashi et al. ${ }^{5}$ retrospectively examined 8 patients with RPILD secondary to JDM, in which 5 of these patients died. Initial thorax $\mathrm{CT}$ findings were found to be the most prevalent subpleural curvilinear shadow, and the others are: ground-glass opacity, pleural effusion, traction bronchiectasis, and consolidation around bronchovascular bundles. It was reported that four of these patients developed air leaks such as pneumomediastinum and pneumothorax during the disease. The retrospective study concluded that the anti-MDA5 antibody level was significantly higher in the RP-ILD patient group. Biopsy or autopsy results of all deceased patients were compatible with the findings of diffuse alveolar damage. ${ }^{5}$ Even though we did not perform any biopsy or autopsy to our patient, the patient's initial thorax CT showed groundglass opacity, atelectasis, subpleural patchy consolidation, pneumomediastinum, and pneumothorax. Intriguingly, the anti-MDA5 antibody was found to be negative in our patient who developed RP-ILD in approximately one and half months after the first symptoms and diagnosis of the disease, and unfortunately, he lost his life shortly after. Sato et al. ${ }^{3}$ examined 29 JDM and JPM patients in their retrospective study and reported that 3 of them developed RP-ILD. While the anti-MDA5 antibodies were positive in 2 of these patients, this antibody was not studied in the other patient. ${ }^{3}$ Bakhshaee et al. $^{6}$ reported a case report of a 21-year-old female patient with RP-ILD secondary to DM presenting with subcutaneous emphysema, pneumomediastinum, and pneumothorax similar to the condition of our patient. In their study, Yeet al. ${ }^{7}$ have reported that pneumothorax or pneumomediastinum has an incidence rate of $8,6 \%$ among ILD secondary to DM/PM. We think that the detection of influenza $B$ in our patient may trigger the development of RP-ILD and may have an additional contribution to the severity of the patient's clinical situation. 
Steroids are the first-line treatment in the acute presentation of ILD secondary to juvenileonset myositis. A 3-day pulse dose steroid and then $1 \mathrm{mg} / \mathrm{kg}$ daily dose of prednisolone is the most preferred treatment in patients with ILD who require hospitalization and whose conditions were suspected to progress into RPILD. The other preferred immunosuppressive agents according to the clinical course of the disease are mycophenolate mofetil, azathioprine, methotrexate, calcineurin inhibitors such as cyclosporine and tacrolimus, cyclophosphamide, hydroxychloroquine, IVIG, and rituximab. ${ }^{1,8,9}$ The two most prevalently used agents in the treatment of RP-ILD are pulse dose steroid and high dose IV cyclophosphamide. According to the literature, it has been reported that rituximab, tofacitinib, TPE are effective in RP-ILD patients who do not respond to standard immunosuppressants. ${ }^{9-13}$ Daily TPE treatment as a rescue therapy was started to our patient who did not respond to pulse dose methylprednisolone, high dose IV cyclophosphamide, and IVIG treatments. Despite 5 sessions of TPE, his lung findings worsened significantly, he was taken to ECMO support and the rituximab treatment was started. The patient who did not respond to all these intensive treatments died on the $35^{\text {th }}$ day of PICU admission. The main reasons why pulsed dose steroid and high dose IV cyclophosphamide treatments were not started immediately after hospitalization to PICU were: the anti-MDA5 antibody result was negative and opportunistic bacterial infections and/or influenza B may have also played a role in the sudden deterioration of the lung findings.

In conclusion, JDM patients should be carefully monitored for the development of ILD. RP-ILD with a high mortality may develop shortly after diagnosis, even if the anti-MDA5 antibody is negative.

\section{Author contribution}

The authors confirm contribution to the paper as follows: study conception and design: OY, MY, CYY, IT; data collection: OY, AA, KB, HY, SŞ; analysis and interpretation of results: $\mathrm{OY}$, EÇ, ÖK; draft manuscript preparation: OY, MY, CYY. All authors reviewed the results and approved the final version of the manuscript.

\section{Conflict of interest}

The authors declare no conflict of interest.

\section{REFERENCES}

1. Schnabel A, Hellmich B, Gross WL. Interstitial lung disease in polymyositis and dermatomyositis. Curr Rheumatol Rep 2005; 7: 99-105.

2. Li D, Tansley SL. Juvenile dermatomyositis-clinical phenotypes. Curr Rheumatol Rep 2019; 21: 74.

3. Sato S, Uejima Y, Nanbu M, et al. Clinical analysis and outcome of interstitial lung disease complicated with juvenile dermatomyositis and juvenile polymyositis. Mod Rheumatol 2017; 27: 652-656.

4. Barut K, Aydin PO, Adrovic A, Sahin S, Kasapcopur O. Juvenile dermatomyositis: a tertiary center experience. Clin Rheumatol 2017; 36: 361-366.

5. Kobayashi N, Takezaki S, Kobayashi I, et al. Clinical and laboratory features of fatal rapidly progressive interstitial lung disease associated with juvenile dermatomyositis. Rheumatology (Oxford) 2015; 54: 784-791.

6. Bakhshaee M, Jokar MH, Mirfeizi Z, Atabati E, Tarighat S. Subcutaneous emphysema, pneumomediastinum and pneumothorax in a patient with dermatomyositis. Iran J Otorhinolaryngol 2017; 29: 113-116.

7. Ye S, Chen XX, Lu XY, et al. Adult clinically amyopathic dermatomyositis with rapid progressive interstitial lung disease: a retrospective cohort study. Clin Rheumatol 2007; 26: 1647-1654.

8. Shappley C, Paik JJ , Saketkoo LA. Myositis-related interstitial lung diseases: diagnostic features, treatment, and complications. Curr Treatm Opt Rheumatol 2019; 5: 56-83. 
9. Schnabel A, Reuter M, Biederer J, Richter C, Gross WL. Interstitial lung disease in polymyositis and dermatomyositis: clinical course and response to treatment. Semin Arthritis Rheum 2003; 32: 273-284.

10. Ogawa Y, Kishida D, Shimojima Y, Hayashi K, Sekijima $Y$. Effective administration of rituximab in anti-MDA5 antibody-positive dermatomyositis with rapidly progressive interstitial lung disease and refractory cutaneous involvement: a case report and literature review. Case Rep Rheumatol 2017; 2017: 5386797.

11. Endo Y, Koga T, Suzuki T, et al. Successful treatment of plasma exchange for rapidly progressive interstitial lung disease with anti-MDA5 antibodypositive dermatomyositis: a case report. Medicine (Baltimore) 2018; 97: e0436.
12. Abe Y, Kusaoi M, Tada K, Yamaji K, Tamura N. Successful treatment of anti-MDA5 antibodypositive refractory interstitial lung disease with plasma exchange therapy. Rheumatology (Oxford) 2020; 59: 767-771.

13. Wendel S, Venhoff N, Frye BC, et al. Successful treatment of extensive calcifications and acute pulmonary involvement in dermatomyositis with the Janus-Kinase inhibitor tofacitinib - A report of two cases. J Autoimmun 2019; 100: 131-136. 